\title{
LoRaWAN Network Performance Test
}

\author{
I. Drotar ${ }^{1, *}$, B. Lukács ${ }^{1}$, M. Kuczmann² \\ ${ }^{1}$ Széchenyi István University, Department of Telecommunications \\ Egyetem Tér 1, H-9026 Győr, Hungary \\ ${ }^{2}$ Széchenyi István University, Department of Automation \\ Egyetem Tér 1, H-9026 Győr, Hungary \\ *E-mail: istvan.drotar@rf.sze.hu
}

Abstract: There are several types of wireless IoT (Internet of Things) networks based on the connection distance between two communicating devices. For covering wide areas, LPWAN (Low Power Wide Area) networks can provide a good solution. These networks provide big coverage and low power consumption. One of the most popular LPWAN network is LoRaWAN (Long Range Wide Area Network). LoRaWAN networks are ideal for sending infrequent, small messages through long distances. In this article the network's capacity, coverage and energy consumption have been tested. These are the most important attributes when designing a LoRaWAN network, so it can satisfy the requirements of LPWAN networks.

Keywords: Low Power Wide Area Network; Internet of Things; Radiowave propagation; Performance analysis

\section{Introduction}

There are more and more electric devices in use, which are capable of sharing information between each other through a network (for example, Wifi, GSM, LoRaWAN, etc.), and we want more services where these devices are required [1]. This trend is true for residential and industrial environments. In industrial use cases, these devices are sensor types most of the time, which monitor or start processes, or they log different values. These devices collect data, which they share between each other, to accelerate processes and increase the efficiency of the infrastructure and resources in factories and companies [2]. This is how the definition of IoT (Internet of Things) was created [3]. 
LPWAN (Low Power Wide Area Network) networks are a special part of IoT networks. These networks main features are small energy consumption and big coverage.

The LoRaWAN network is a LPWAN network, that is mainly used to uplink messages, and the messages are small in size [4]. LoRaWAN devices are usually battery powered, and they use wireless messages to send tata towards the network. Gateways collect the messages sent by LoRaWAN devices and transmit it towards the server. LoRaWAN protocol was created by the LoRa Alliance, which is a nonprofit association with more than 500 member companies. One of the founding members of the LoRa Alliance is Semtech, which developed the LoRa modulation, and contributed to the LoRaWAN protocol. Semtech is also producing high performance semiconductors. A thorough description and explanation of the LoRaWAN architecture and protocol can be seen in [5].

LoRaWAN networks use unlicensed ISM frequency bands, for example, in Europe LoRaWAN networks operate in the $868 \mathrm{MHz}$ ISM band (the maximum output power is $14 \mathrm{dBm}$ in this band, and the duty cycle must be less or equal to $0.1 \%$ if the device does not use listen before talk mechanism). It uses a spread spectrum technology, that is why it is more resilient against narrow band interferences. Thanks to the spread spectrum technology, its power spectral density is much smaller than other technologies, and it can be even below the noise level, thereby devices outside of the network may not even be able to sense the operation of the LoRaWAN network. The LoRaWAN devices use LoRa modulation. This is a slower data speed modulation, but the signals can be received with low signal strengths and the power consumption of the modulation is low as well. In the article the main properties of the LoRaWAN network, which are the capacity, coverage and power consumption were tested, because in the future it is expected that one gateway will have to manage tens of thousands of LoRaWAN devices, it has to cover a big area and the devices will have to work with a battery for a long time (even 10 years).

LoRa GPS motes were used, which contain the RN2483 LoRaWAN module. The gateways used are Kerlink Wirnet Stations. These gateways can receive messages at the same frequency and at the same time, if the data rate values of the transmissions are different.

The performance of the LoRaWAN devices highly depend on the settings [6]. In this case study the LoRaWAN devices were tested with the most commonly used settings. The used frequency band is $868 \mathrm{MHz}$, the bandwidth of the devices is 125 $\mathrm{kHz}$ and the data rate is between 0 and 5 .

Compared to the capacity test in [7] where $~ 40 \%$ packet loss was reached at around 500 devices (with data rate 0 setting), in our experiments this packet loss 
ratio was reached with around 9600 simulated devices. The difference may be caused by different parameter settings or the different approach of the experiments, this could be a basis for further investigations.

\section{LoRa modulation}

The LoRa modulation uses CSS (Chirp Spread Spectrum) spread spectrum technology, which codes the information with linearly changing chirp impulses [8]. A chirp is a sinus signal, which's frequency is increasing or decreasing over time. If the frequency is increasing, we call it upchirp, and if the frequency is decreasing, we call it downchirp. A LoRa symbol occupies the whole bandwidth, and the information is coded in the sudden frequency changes in the symbol.

A LoRa symbol consists of chirps. The number of chirps generated per second is equal to the bandwidth. The spreading factor $(S F)$ determines that how many bits and chirps a symbol contains.

$$
1 \text { symbol }=2^{S F} \text { chirp, }
$$

If we increase the value of $S F$ by 1 , then the number of bits contained by the symbol increases, but the chirp/second stays the same, and the number of chirps that a symbol contains doubles. This results in that the time needed to send the same message with one higher $S F$ value, will be doubled, but the data speed will almost be half of the one lower $S F$ value. In turn for the lower data speed, the receiver sensitivity will be higher, and messages with lower signal strength can be received and decoded.

In the LoRaWAN protocol the $S F$ value can be changed by the data rate value (DR). The data rate 0 is the slowest LoRa modulation $(S F=12)$, and data rate 5 is the fastest $(S F=7)$, with $125 \mathrm{kHz}$ bandwidth. In LoRaWAN protocol, devices can change their data rate settings with adaptive data rate (ADR) to best adapt to the environment it is operating in [9].

\section{Capacity test}

\subsection{Measurement method}

In Europe the LoRaWAN networks operate in the $868 \mathrm{MHz}$ ISM band. This frequency band can be split into channels according to different patterns. The Semtech channel allocation can be seen in Table 1. 
Table 1. Semtech LoRaWAN frequency allocation

\begin{tabular}{|c|c|c|c|c|}
\hline Channel & $\begin{array}{c}\text { Frequency } \\
{[\mathbf{M H z}]}\end{array}$ & $\begin{array}{c}\text { Bandwidth } \\
{[\mathbf{k H z}]}\end{array}$ & DR & Modulation \\
\hline 0 & 868.1 & 125 & $0-5$ & LoRa \\
\hline 1 & 868.3 & 125 & $0-5$ & LoRa \\
\hline 2 & 868.5 & 125 & $0-5$ & LoRa \\
\hline 3 & 867.1 & 125 & $0-5$ & LoRa \\
\hline 4 & 867.3 & 125 & $0-5$ & LoRa \\
\hline 5 & 867.5 & 125 & $0-5$ & LoRa \\
\hline 6 & 867.7 & 125 & $0-5$ & LoRa \\
\hline 7 & 867.9 & 125 & $0-5$ & LoRa \\
\hline LoRa & 868.3 & 125 & 6 & LoRa \\
\hline FSK & 868.8 & 125 & 7 & FSK \\
\hline RX2 & 869.525 & 125 & 0 & LoRa \\
\hline
\end{tabular}

A LoRaWAN gateway can receive messages simultaneously with different data rate values on a single frequency. This means that one physical channel, where 6 data rate values are enabled (0-5) is equal to six logic channels. There is no synchronization between devices, so if a gateway receives two messages at the same time on the same logic channel, then the messages collide, and the data is lost. From the IoT viewpoint it is critical how many devices a network can support, how many device's data can it collect without severe data loss.

Since there were not enough devices available to test the capacity, multiple logical devices were simulated with a single physical device through settings. The LoRa GPS motes will only use one logic channel. The duty cycle must be set to $100 \%$ on the devices, otherwise the LoRaWAN module blocks the rf output until the set duty cycle value is met. Furthermore, the ADR option must be turned off as well, so the devices don't change their data rate settings, and all the devices were set to the same data rate value.

The LoRaWAN device test parameters:

- Frequency: $868 \mathrm{MHz}$

- Tx Power: $14 \mathrm{dBm}$

- Data rate: $0-5$

- Bandwidth: $125 \mathrm{kHz}$

- Coding rate: $4 / 5$

The LoRa GPS motes sent unconfirmed messages (the mote does not wait for a confirmation reply from the gateway). Every mote waits a random time between 48 seconds after sending a message. This way a mote sends a message in every 6 
seconds on average. The motes sent the exact same payload, which is the following HEX character string: “"00112233445566778899”.

The benchmark is a LoRaWAN device that sends a message every hour. The tested devices were compared to this benchmark device. The number of logic channels were narrowed down to one for the tested devices, this gives a multiplication factor of eight. The tested devices sent a message every six seconds, on average, this gives another multiplication factor of 600. If the effects of the settings are summarized, one of the tested devices is equal to 4800 benchmark devices. With this, we can simulate packet collision with few devices.

The devices were placed into the fully unechoing chamber of Széchenyi István University's Radio Frequency Test Laboratory. The messages sent by the LoRaWAN devices were transmitted through a test antenna inside the chamber to the LoRaWAN gateway, outside of the chamber. With this setup, there was no interference from the outside. This was needed to get an interference free measurement, because a lot of other wireless devices use the $868 \mathrm{MHz}$ ISM band.

The received messages are stored in a SQL database by a server. Every LoRa Mote device numbers their transmitted messages, and with analyzing the stored messages, we can determine how many messages were lost during the test.

\subsection{Measurement results}

The test was carried out with data rate 0 and 5 settings, and with 2, 4 and 8 devices. The results can be seen in Figure 1. The importance of the data rate value from the capacity viewpoint can be seen from the results. With 38400 simulated devices, and with data rate $5,11.56 \%$ of messages were lost. This ratio increased when data rate was changed to 0 , and $90.56 \%$ of messages were lost.

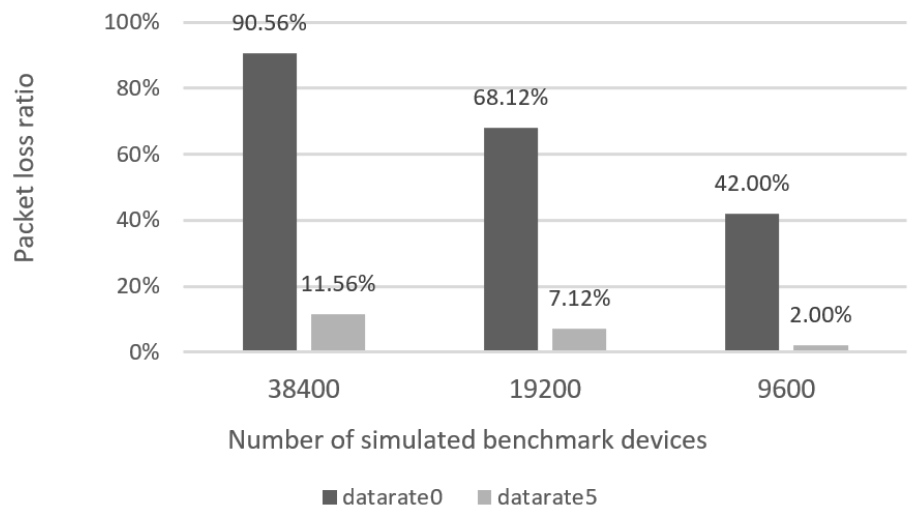

Figure 1. Packet loss ratio with different data rate settings 
Based on these results, theoretically tens of thousands of LoRaWAN devices can operate within a single gateway's area, if the devices are similar to the benchmark device. These results depend on the data size and the time between two consecutive messages. Also, no interference from the environment was present during the test, which would increase the packet loss ratio in a real use case scenario.

For increasing channel capacity, a suitable data rate selection algorithm should be implemented into LoRaWAN nodes. An example for data rate selection algorithms can be seen in [10].

\section{Coverage test}

\subsection{Measurement method}

As a LPWAN network, a LoRaWAN gateway must cover a big area. To test the coverage of a gateway, we must collect data on the area, to be covered. With the collected data we can tune wave propagation models to analyze and simulate the coverage of the LoRaWAN gateway. During the test, the signal strength of the uplink messages were saved, because the system is designed for this direction of communication.

The test was carried out in Györ, Hungary (a city with approximately 130000 inhabitant). We used a single LoRaWAN node, that sent its GPS coordinates out, while moving around the different parts of the city. Four LoRaWAN gateways were receiving the message, storing the RSSI value as well. A sample of collected data can be seen in Figure 2.

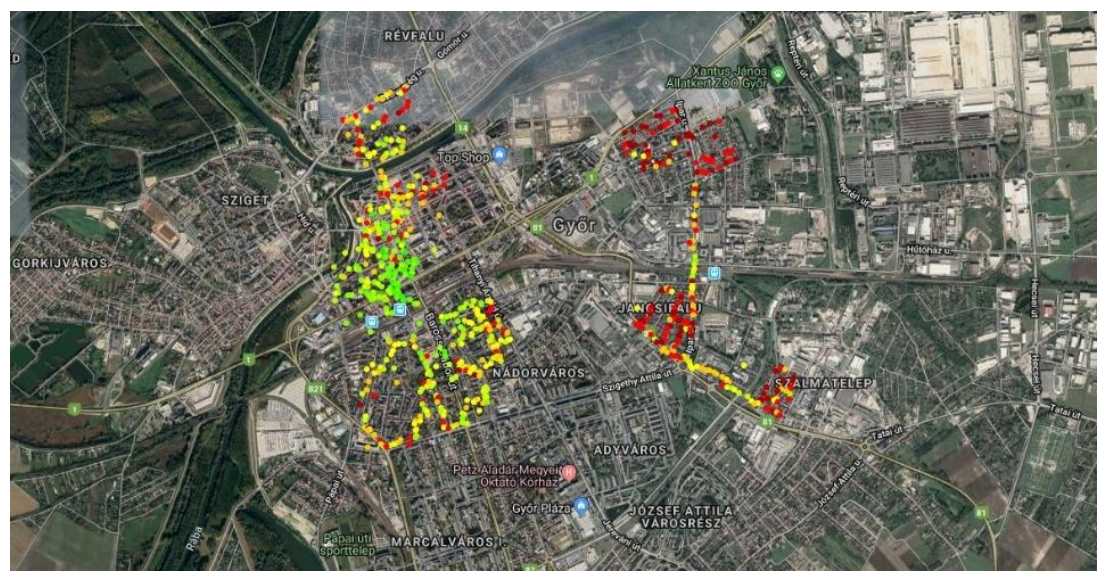

Figure 2. Collected data for coverage measurement in Györ 
For data collection, a LoRa GPS mote, three LoRaWAN gateway and an application server was used. The LoRa GPS Mote sent a message with its location information every 10 seconds. We know how strong electromagnetic field the LoRa GPS mote creates at the gateway, because the gateways store the received signal strength for every received message.

The gateways forward the message, and additional data to a NodeJS server, which stores the data in a SQL database, and it can also visualize the received data on an online interface.

To design a LoRaWAN network, we must be able to simulate the area covered by gateways. For this, three of the most commonly used empiric wave propagation models were used. These models were used, because they are commonly used for WAN network design, and they are easy to use, especially if there is not too much data available about the environment. The tested wave propagation models [11][12][13].

\subsubsection{Okumura}

$$
L=L F+A_{M U}-G_{\text {hte }}-G_{\text {hre }}-G_{\text {area }}
$$

$L$ is the calculated attenuation $(\mathrm{dB}), L F$ is the Free space attenuation $(\mathrm{dB}), A_{M U}$ is the Medium attenuation relative to free space $(\mathrm{dB}), G_{h t e}$ is the gain factor of the base station $(\mathrm{dB}), G_{\text {hre }}$ is the gain factor of the mobile station $(\mathrm{dB})$, and $G_{\text {area }}$ is the environment's correction gain $(\mathrm{dB})$.

\subsubsection{Hata (Urban environment)}

$$
\begin{aligned}
& L_{u}=69.55+26.16 \log _{10}(f)-13.82 \log _{10}\left(h_{b}\right)-C_{H}+[44.9- \\
& \left.6.55 \log _{10}\left(h_{b}\right)\right] \log _{10}(d)
\end{aligned}
$$

$L_{u}$ is the attenuation in urban environment $(\mathrm{dB}), h_{b}$ is the height of the base station antenna $(\mathrm{m}), h_{m}$ is the height of the mobile station antenna $(\mathrm{m}), C_{H}$ is the correction factor of mobile station antenna $(\mathrm{dB}), f$ is the used frequency $(\mathrm{MHz})$, and $d$ is the distance $(\mathrm{km})$.

Value of $C_{H}$ in urban environment:

$$
C_{H}=0.8+\left[1.1 \log _{10}(f)-0.7\right] h_{m}-1.56 \log _{10}(f)
$$

\subsubsection{COST-Hata}

$$
\begin{aligned}
& L=46.3+33.9 \log _{10}(f)-13.82 \log _{10}\left(h_{b}\right)-a\left(h_{r}, f\right)+[44.9- \\
& \left.6.55 \log _{10}\left(h_{b}\right)\right] \log _{10}(d)+C
\end{aligned}
$$

Value of $\left(h_{r, f}\right)$ : 


$$
\left[1.1 \log _{10}(f)-0.7\right] h_{r}-\left[1.56 \log _{10}(f)-0.8\right]
$$

$L$ is the calculated attenuation $(\mathrm{dB}), f$ is the used frequency $(\mathrm{MHz}), h_{b}$ is the height of the base station antenna $(\mathrm{m}), h_{r}$ is the height of the mobile station antenna $(\mathrm{m})$, $a\left(h_{r}, f\right)$ is the antenna correction factor of the mobile station $(\mathrm{dB})$. If the environment is urban, then the correction factor described in the Hata model is to be used.

The Value of $C$ is $0 \mathrm{~dB}$ in small and medium cities, and $3 \mathrm{~dB}$ in big cities.

\subsection{Measurement results}

For all three wave propagation models, the average and the deviation of the difference of the calculated and measured values are used for optimisation. The average difference can be corrected with a constant value. The standard deviation defines the accuracy of the wave propagation model. The models are corrected with the calculated constant values, to make the coverage simulation as accurate as possible. The lower the standard deviation, the more accurate the wave propagation model is.

For every measurement point, the difference between the measured value (measured by the gateway) and the simulated value (simulated with propagation models) is calculated. With these difference values, the average and standard deviation values were calculated. The average difference values were calculated with Eq. 7, and the standard deviation values were calculated with Eq. 8.

$$
\begin{aligned}
\mu & =\frac{1}{N} \sum_{i=1}^{N}\left(x_{i}-y_{i}\right) \\
\sigma & =\sqrt{\frac{1}{N} \sum_{i=1}^{N}\left(Z_{i}-\mu\right)}
\end{aligned}
$$

In the equations above, $\mu$ is the average difference value, $x_{i}$ is the simulated field strength, $y_{i}$ is the measured field strength, $\sigma$ is the standard deviation and $z_{i}$ is the difference between the simulated and the measured field strength. The calculated average difference and standard deviation values can be seen in Hiba! A hivatkozási forrás nem található.Table 2 .

Table 2. Average difference and standard deviation values of tested wave propagation models

\begin{tabular}{|c|c|c|c|}
\hline & Okumura & Hata & COST-Hata \\
\hline $\begin{array}{c}\text { Average } \\
\text { difference }\end{array}$ & $1.61 \mathrm{~dB}$ & $-6.69 \mathrm{~dB}$ & $-6.17 \mathrm{~dB}$ \\
\hline $\begin{array}{c}\text { Standard } \\
\text { deviation }\end{array}$ & $6.75 \mathrm{~dB}$ & $6.88 \mathrm{~dB}$ & $6.88 \mathrm{~dB}$ \\
\hline
\end{tabular}


Since the standard deviation of the Okumura model was the lowest, this wave propagation model is the most accurate out of the three. Because of this, the Okumura model was used for coverage simulation.

\subsection{Coverage results}

The only additional thing needed is the link budget, to calculate the coverage. The link budget determines the maximum attenuation, at which the gateway is still able to receive and decode an uplink message. The link budget was calculated based on the equations below:

$$
\begin{gathered}
\text { Link budget }=P_{t x}+G_{t x}+G_{r x}+\left|R S S I_{\min }\right| \\
\text { Link budget }=14-0.62-2.7+125
\end{gathered}
$$

After calculating, the link budget is $141.08 \mathrm{~dB}$. If this attenuation value is put into the wave propagation equations, we get the theoretical distance, where the maximum attenuation is present, where the gateway is still able to receive an uplink message. Unfortunately, it is not that easy, as we can see from the standard deviation values as well. The main cause of wave propagation inaccuracy is fading. To compensate the fading effect, the standard deviation values are used for fading reserve. Standard deviation is assumed, so if the standard deviation values are used as reserve, the probability that the attenuation will be lower, than the calculated attenuation is $68 \%$. This probability increases to $95 \%$, if the standard deviation's doubled value is used. We do not care about the case, where the attenuation is smaller than the calculated, since that does not cause problems for the communication.

If the standard deviation's doubled value is the fading reserve, then the corrected link budget is $127.58 \mathrm{~dB}$ for the Okumura model, and $127.32 \mathrm{~dB}$ for the Hata and COST-Hata models. Substituting the corrected link budget values into the wave propagation models gives us the maximum distance, where the gateway is able to receive uplink messages, with a probability of $95 \%$. The maximum distance of the wave propagation models can be seen in Table 3, when the fading reserve is the doubled value of the standard deviation.

Table 3. Maximum calculated distance with using the standard deviation's doubled value

\begin{tabular}{|c|c|c|c|}
\hline & Okumura & Hata & COST-Hata \\
\hline $\begin{array}{c}\text { Maximum } \\
\text { distance }\end{array}$ & $1700 \mathrm{~m}$ & $1810 \mathrm{~m}$ & $1800 \mathrm{~m}$ \\
\hline
\end{tabular}

If the fading reserve is equal to the standard deviation, the link budget is 134.33 $\mathrm{dB}$ for the Okumura model, and $134.32 \mathrm{~dB}$ for Hata and COST-Hata models. The maximum distances with the standard deviation's value can be seen in Table 4. 


\section{Table 4. Maximum calculated distance using the standard deviation's value}

\begin{tabular}{|c|c|c|c|}
\hline & Okumura & Hata & COST-Hata \\
\hline $\begin{array}{c}\text { Maximum } \\
\text { distance }\end{array}$ & $3300 \mathrm{~m}$ & $2790 \mathrm{~m}$ & $2850 \mathrm{~m}$ \\
\hline
\end{tabular}

The maximum distances got a lot bigger, but at the maximum distance, the probability of a successful transmission is $68 \%$. In this case, the network should be designed that there are always multiple gateways available, so even if a gateway fails to receive a message, the other gateways may be able to, thus lowering the risk of losing messages.

\section{Energy consumption}

\subsection{Measurement method}

In a lot of IoT use cases, wired power supply is not an option, batteries must be used, and these devices are expected to work for years, sometimes even 10 years. To achieve this long battery life, energy consumption is crucial [14].

The device that was tested is only a demo device, that is why its power consumption is not optimized, but the effect of different settings can be still measured. The device has a $3.7 \mathrm{~V}, 750 \mathrm{mAh}$ battery. The device was measured with different configurations [15]. The measurement was taken with a multimeter, and a data recording software. The software queried the current input of the device and calculated the power from that in real time.

The device uses $125 \mathrm{kHz}$ bandwidth at the $868 \mathrm{MHz}$ band, with 4/5 coding rate.

\subsection{Measurement results}

First the sleep mode was measured, since these devices will spend most of their times in this mode. In sleep mode the device draws $0.45 \mathrm{~mA}$. With this current, the device can work for 69.44 days. With industrial devices, the drawn current can be under $100 \mathrm{uA}$ in sleep mode, which can multiply the battery life of the device.

For a device to send a message with data rate 0 setting, with $14 \mathrm{dBm}$ radiated power, it needs about $0.59583 \mathrm{~J}$. If the device sends 1 message every day, the battery life reduces to 69.16 days. This also shows, that with these kinds of devices, the most important mode is the sleep mode. After this, the radiated power and the data rate settings' effect on power consumption were tested. With every setting, the device transmitted the same message. 
Table 5. $\quad$ Power needed for transmitting a single message with different power and data rate settings

\begin{tabular}{|c|c|c|c|c|c|}
\hline DR/PWR & $\begin{array}{c}\mathbf{1 1 . 0 9} \\
\mathbf{d B m}\end{array}$ & $\begin{array}{c}\mathbf{8 . 0 6} \\
\mathbf{d B m}\end{array}$ & $\begin{array}{c}\mathbf{4 . 4 2} \\
\mathbf{d B m}\end{array}$ & $\begin{array}{c}\mathbf{0 . 9 3} \\
\mathbf{d B m}\end{array}$ & $\mathbf{- 2 . 4} \mathbf{~ d B m}$ \\
\hline 0 & $100.0 \%$ & $98.7 \%$ & $90.1 \%$ & $89.4 \%$ & $71.3 \%$ \\
\hline 1 & $67.1 \%$ & $66.2 \%$ & $60.4 \%$ & $60.0 \%$ & $47.8 \%$ \\
\hline 2 & $39.5 \%$ & $39 \%$ & $35.6 \%$ & $35.3 \%$ & $28.2 \%$ \\
\hline 3 & $32.2 \%$ & $31.8 \%$ & $29.1 \%$ & $28.8 \%$ & $23.1 \%$ \\
\hline 4 & $23.2 \%$ & $22.9 \%$ & $20.9 \%$ & $20.7 \%$ & $16.5 \%$ \\
\hline 5 & $15.4 \%$ & $15.2 \%$ & $13.9 \%$ & $13.8 \%$ & $11.0 \%$ \\
\hline
\end{tabular}

The most power is needed when the data rate is 0 , and the radiated power is 11.09 $\mathrm{dBm}$. The power needed to send a message in this setting represents $100 \%$, and other power consumptions are compared to this. Increasing the data rate value lowers the power consumption significantly, and it is also favorable from a capacity viewpoint. Lowering the radiated power does not lower the power consumption as much. Furthermore, lowering the radiated power can cause the device to lower its data rate value, because the gateway cannot receive the message due to attenuation, so the device can lower its data rate to increase the receiver sensitivity. Lowering the radiated power can even cause to increase power consumption, and it can also be bad for capacity. If the transmit power is decreased, it is possible that the gateway is no longer able to decode the messages. In this case the LoRaWAN node needs to decrease its data rate setting, which increases the transmit time and the power consumption. The increased transmit time also increases the chance of messages colliding, increasing the packet loss ratio.

\section{Summary}

LoRaWAN gateways should be placed the way that LoRaWAN devices can operate in the highest data rate option possible. In data rate 5 , the chance of packet collision can be $\sim 80 \%$ lower, than in data rate 0 . The capacity of lower data rate logic channels is much bigger, and if the network wants to utilize the maximum capacity, gateways should be placed close enough to each other, to let devices use lower data rate channels.

A smaller city (like Györ, population is $\sim 130$ thousand) can be covered with three gateways, it provides sufficient coverage, and transmissions are possible from several kilometers away in urban environment.

The higher data rate is also beneficial for energy consumption. It is more effective in lowering energy consumption, than lowering transmission power. With changing the data rate to 0 from 5 , the power needed for the transmission is lowered by $\sim 85 \%$. 
While changing the output power from $11.09 \mathrm{dBm}$ to $-2,4 \mathrm{dBm}$ only yields a $29 \%$ decrease in energy consumption. In order to get the longest operation time with a single charge, the LoRaWAN device should transmit with the highest possible data rate.

\section{References}

[1] H. N. Saha, A. Mandal, A. Sinha, Recent trends in the Internet of Things, in: 2017 IEEE 7th Annual Computing and Communication Workshop and Conference (CCWC), 2017. doi: https://doi.org/10.1109/CCWC.2017.7868439

[2] P. Sethi, S. R. Sarangi, Internet of Things: Architectures, Protocols, and Applications, Journal of Electrical and Computer Engineering 2017 (2017) pp. 1-25.

doi: https://doi.org/10.1155/2017/9324035

[3] K. K. Patel, S. M. Patel, Internet of Things-IOT: Definition, Characteristics, Architecture, Enabling Technologies, Application \& Future Challenges, International Journal of Engineering Science and Computing 6 (5) (2016) pp. $6122-6131$.

[4] D. M. Ibrahim, Internet of Things Technology based on LoRaWAN Revolution, in: 2019 10th International Conference on Information and Communication Systems (ICICS), 2019 pp. 234-237. doi: https://doi.org/10.1109/iacs.2019.8809176

[5] M. A. Ertürk, M. A. Aydin, M. T. Büyükakkslar, A Survey on LoRaWAN Architecture, Protocol and Technologies, Future Internet 11 (10) (2019) p. 216.

doi: https://doi.org/10.3390/fi11100216

[6] R. Harwahyu, A. Presekal, R. F. Sari, LoRaWAN Performance Evaluation with Optimized Configuration, International Journal of Future Generation Communication and Networking 11 (4) (2018) pp. 51-68.

doi: http://dx.doi.org/10.14257/ijfgen.2018.11.4.05

[7] M. C. Bor, U. Roeding, T. Voigt, Do LoRa Low-Power Wide-Area Networks Scale?, in: Proceedings of the 19th ACM International Conference on Modeling, Analysis and Simulation of Wireless and Mobile Systems: 2016, pp. 59-67.

doi: https://doi.org/10.1145/2988287.2989163 
[8] M. Chiani, A. Elzanaty, On the LoRa Modulation for IoT: Waveform Properties and Spectral Analysis, IEEE Internet of Things Journal 6 (5) (2019) pp. 8463-8470.

doi: https://doi.org/10.1109/jiot.2019.2919151

[9] LoRa Alliance Inc., LoRaWAN ${ }^{\mathrm{TM}} 1.1$ Specification, 2017.

URL https://Iora-alliance.org/sites/default/files/201804/lorawantm_specification_-v1.1.pdf

[10] A. Farhad, D. Kim, J. Pyun, Resource Allocation to Massive Internet of Things in LoRaWANs, Sensors 20 (9) (2020) p. 2655.

doi: https://doi.org/10.3390/s20092645

[11] P. Keawbunsong, P. Supanakoon, S. Promwong, Hata's Path Loss Model Calibration for Prediction DTTV Propagation in Urban Area of Southern Thailand, IOP Conference Series: Materials Science and Engineering 83 (2015).

doi: https://doi.org/10.1088/1757-899x/83/1/012013

[12] ITU, Handbook - Terrestrial land mobile radiowave propagation in the VHF/UHF bands, Geneva, 2002.

URL http://www.itu.int/net4/ITUT/registration/Resolver/Index?handle_id=11.1002/pub/800c9 4ad-en

[13] N. S. Nkordeh, A.A.A Atayero, LTE Network Planning using the HataOkumura and the COST-231 Hata Pathloss Models, in: World Congress on Engineering (WCE) 1 (2014).

[14] B. Martinez, M. Montón, The Power of Models: Modeling Power Consumption for IoT devices, IEEE Sensors Journal 15 (10) (2015) pp. 5777 5789.

doi: https://doi.org/10.1109/jsen.2015.2445094

[15] D. Magrin, M. Capuzzo, A. Zanella, A through Study of LoRaWAN Performance Under Different Parameter Settings, IEEE Internet of Things Journal 7 (1) (2020) pp. 116-127.

doi: https://doi.org/10.1109/jiot.2019.2946487 